\title{
Encounters with Di-Baryons - from the ABC Effect to a New Resonance?
}

\author{
Elena Perez del Rio*, M. Bashkanov, H. Clement, E. Doroshkevich, A. Pricking, T. \\ Skorodko, G. J. Wagner, for the WASA-at-COSY collaboration \\ Physikalisches Institut der Universität Tübingen, Germany \\ E-mail: perezapit.physik.uni-tuebingen.de
}

The ABC effect, an intriguing low-mass enhancement in the $\pi \pi$ invariant mass spectrum, is known from inclusive measurements of two-pion production in nuclear fusion reactions to the few-body systems d, ${ }^{3} \mathrm{He}$ and ${ }^{4} \mathrm{He}$. It was first observed 1960 by Abashian, Booth and Crowe in the inclusive $p d \rightarrow{ }^{3} \mathrm{He} \mathrm{X}$ reaction. Its explanation has been a puzzle since then. In an effort to solve this long-standing problem by exclusive and kinematically complete high-statistics experiments, we have measured the fusion reactions to $\mathrm{d},{ }^{3} \mathrm{He}$ and ${ }^{4} \mathrm{He}$ with WASA-at-COSY. These measurements cover the full energy region, where the $\mathrm{ABC}$ effect has been observed previously in inclusive reactions. In a recent kinematically complete measurement of the $p n \rightarrow d \pi^{0} \pi^{0}$ reaction we have shown that the $\mathrm{ABC}$ effect in this basic double-pionic fusion reaction is correlated with a narrow structure in the total cross section with quantum numbers $I\left(J^{P}\right)=0\left(3^{+}\right)$, a mass of 2.37 $\mathrm{GeV}$ and a width of about $70 \mathrm{MeV}$. The mass is about $90 \mathrm{MeV}$ below 2 times the mass of $\Delta$, the mass of a $\Delta \Delta$ system, and the width is three times narrower than expected from a conventional $\mathrm{t}$-channel $\Delta \Delta$ process. In the double-pionic fusion reaction to the helium isotope $d d \rightarrow{ }^{4} \mathrm{He} \pi^{0} \pi^{0}$ again the $\mathrm{ABC}$ effect is observed to be correlated with the appearance of a resonance-like structure in the total cross section at the same excess energy. From a previous exclusive experiment at CELSIUS-WASA it is known that the double-pionic fusion to ${ }^{3} \mathrm{He}$ also exhibits a pronunced $\mathrm{ABC}$ effect. New data from COSY on the $p d \rightarrow{ }^{3} \mathrm{He} \pi^{0} \pi^{0}$ reaction scanning the full $\mathrm{ABC}$ region are presented as well as the status of measurements in other reaction channels, where the new resonance might contribute.

51st International Winter Meeting on Nuclear Physics

21-25 January 2013

Bormio (Italy)

* Speaker. 


\section{Introduction}

The $\mathrm{ABC}$ effect, an intriguing low-mass enhancement in the $\pi \pi$ invariant mass spectrum, has first been observed in inclusive measurements of the $p d \rightarrow{ }^{3} \mathrm{He} \mathrm{X}$ reaction by Abashian, Booth and Crowe [1]. Subsequent bubble-chamber [2,3] and single-arm magnetic spectrometer measurements $[4,5,6,7,8,9,10,11]$ suggested this enhancement to be correlated with the production of an isoscalar pion pair in fusion reactions to light nuclei. Its explanation has been a puzzle since more than 50 years. Therefore it has been named just after the initials of those authors, who first observed this effect.

In recent exclusive and kinematically complete measurements of the $p n \rightarrow d \pi^{0} \pi^{0}$ reaction it has been demonstrated $[12,13,22]$ that the $\mathrm{ABC}$ effect in this basic double-pionic fusion reaction is correlated with a narrow structure in the total cross section with quantum numbers $I\left(J^{P}\right)=0\left(3^{+}\right)$, a mass of $2.37 \mathrm{GeV}$ and a width of about $70 \mathrm{MeV}$. The mass is about $90 \mathrm{MeV}$ below $2 m_{\Delta}$, the mass of a $\Delta \Delta$ system and the width is three times narrower than expected from a conventional $t$-channel $\Delta \Delta$ process.

\section{Experiments}

In an effort to solve this long-standing problem by exclusive and kinematically complete highstatistics experiments, we have measured the fusion reactions to $\mathrm{d},{ }^{3} \mathrm{He}$ and ${ }^{4} \mathrm{He}$ with WASA at COSY. These measurements cover the full energy region, where the $\mathrm{ABC}$ effect has been observed previously in inclusive reactions. They complement the systematic two-pion production measurements carried out at PROMICE/WASA [15, 16, 17], CELSIUS/WASA [13, 18, 19, 20, 21, 27, 28], COSY-TOF [23, 24], COSY-MOMO [29], WASA-at-COSY [25, 30, 31] and COSY-ANKE [26].

The bulk of these experiments has been performed with the WASA detector featuring a solid angle coverage of nearly $4 \pi$, windowless hydrogen and deuterium pellet target systems $[32,33]$ as well as a reliable particle identification for $\gamma, \pi, p, n, d,{ }^{3} \mathrm{He}$ and ${ }^{4} \mathrm{He}$ ejectiles.

Measurements at WASA have been performed by detection of all ejectiles of an event (with the exception of spectator nucleons) allowing thus kinematic fits with several overconstraints in the data analysis. The $p n$ initiated reactions have been studied via the quasifree $p d$ process with a spectator proton resulting from the target deuteron. By use of the Fermi motion of the active nucleon in the deuteron target the energy dependence of $p n$ and $p p$ initiated reactions could be measured over a range of more than $100 \mathrm{MeV}$ with just a single beam energy. In case of the fusion reaction to ${ }^{3} \mathrm{He} p d$ initiated reactions have been measured, either by hitting a deuteron target with a proton or a deuteron beam. The latter gives rise to quasifree $p d$ collisions and through the Fermi motion gives acess to a broad region of energy, where the $\mathrm{ABC}$ effect is expected to take place.

\section{Discussion of Results}

From these measurements total as well as differential cross sections have been obtained over the full energy region, where the $\mathrm{ABC}$ effect has been found previously.

As an example we show in Figs. 1 and 2 the Dalitz plots for two different energies as well as its projections for the double pionic fusions to $\mathrm{d}$ and ${ }^{4} \mathrm{He}$, respectively [12, 30, 31]. 

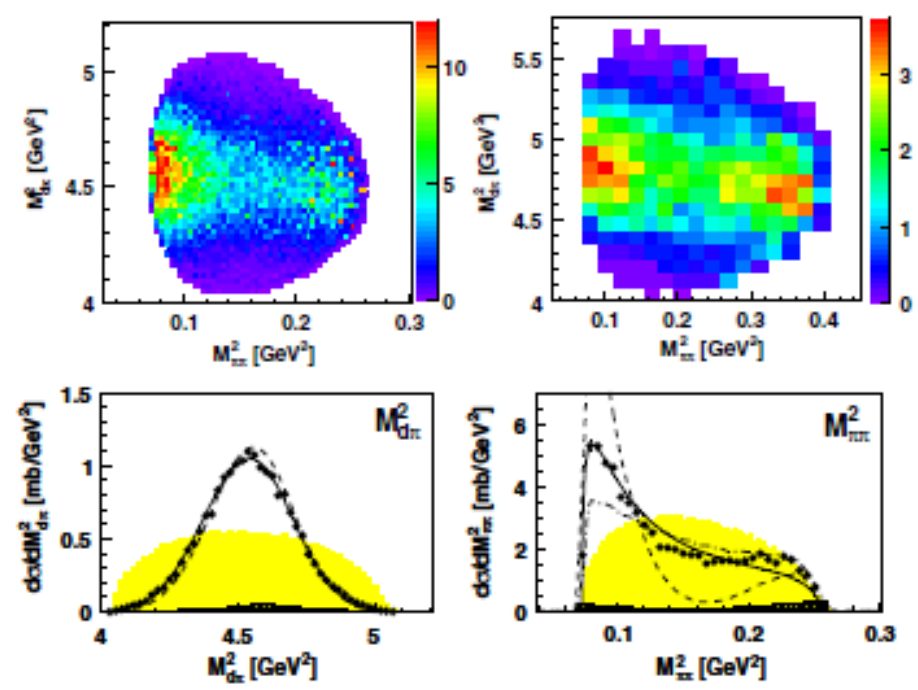

Figure 1: Top: Dalitz plots of $\mathrm{M}^{2} d \pi^{0}$ versus $\mathrm{M}^{2} \pi^{0} \pi^{0}$ at $\sqrt{s}=2.38 \mathrm{GeV}$ (peak cross section) (left) and at $\sqrt{s}=2.5 \mathrm{GeV}$ (right). Bottom: Dalitz plot projections $\mathrm{M}^{2}{ }_{4} \mathrm{He}^{0}$ (left) and $\mathrm{M}^{2} \pi^{0} \pi^{0}$ (right) axes at $\sqrt{s}=2.38$ $\mathrm{GeV}$. The curves denote calculations for a $s$-channel resonance decaying into $\Delta \Delta$ with $J^{P}=3^{+}$with (solid) and without (dash-dotted) form factor as well as for $J^{P}=1^{+}$(dashed). Hatched and shaded areas represent systematic uncertainties and phase-space distributions, respectively. From Ref. [12],Fig. 4.


Figure 2: Top: Dalitz plot of $\mathrm{M}^{2}{ }_{4}{ }_{H} \pi^{0}$ versus $\mathrm{M}^{2}{ }_{\pi^{0}} \pi^{0}$ for data (left) at the energy of the peak cross section $(\sqrt{s}=4.24 \mathrm{GeV})$ and Monte Carlo calculation for an $s$-channel pn resonance with $m=2.37$ and $\Gamma=124$ $\mathrm{MeV}$ including Fermi morion of the nucleons in the initial and final nuclei. Bottom: Distributions of $\mathrm{M}_{4} \mathrm{He}^{0}$ (left) and $\mathrm{M}_{\pi^{0} \pi^{0}}$ (right) at $\sqrt{s}=2.27 \mathrm{GeV}$. The shaded areas represent the phase-space distributions. Dotted lines give $t$-channel $\Delta \Delta$ calculations, whereas the solid line shows a calculation of a $p n s$-channel resonance for illustration. From Ref. [30, 31], Figs. 2 and 3. 
In both cases we observe a pronounced $\mathrm{ABC}$ effect, i.e. low-mass enhancement - similar to the situation in the $p d \rightarrow{ }^{3} \mathrm{He} \pi^{0} \pi^{0}$ reaction [27]. This experimental finding strongly contravenes conventional calculations based on the mutual excitation of the two colliding nucleons to their $\Delta(1232)$ state by $t$-channel meson exchange, which rather predict a gentle two-hump structure relative to phase space $[12,20,30]$.

The basic double-pionic fusion to deuterium features three different reaction channels:

- $p n \rightarrow d \pi^{0} \pi^{0}$, which is purely isoscalar

- $p p \rightarrow d \pi^{+} \pi^{0}$, which is purely isovector, and

- $p n \rightarrow d \pi^{+} \pi^{-}$, which contains both isoscalar and isovector contributions.

They are connected by the following isospin relation [34] for their total cross sections:

$$
\sigma\left(p n \rightarrow d \pi^{+} \pi^{-}\right)=2 \sigma\left(p n \rightarrow d \pi^{0} \pi^{0}\right)+\frac{1}{2} \sigma\left(p p \rightarrow d \pi^{+} \pi^{0}\right),
$$

which allows for a cross check on the internal consistency of measurements.

Based on our measurements of all three reactions we have carried out an isospin decomposition of the isospin-mixed $p n \rightarrow d \pi^{+} \pi^{-}$reaction by use of both the isovector $p p \rightarrow d \pi^{+} \pi^{0}$ and the isoscalar $p n \rightarrow d \pi^{0} \pi^{0}$ channel. We find internal consistency of the measurements within their uncertainties. The result of the isospin decomposition is shown in Fig. 3 [22, 35].

The basic isovector fusion process $p p \rightarrow d \pi^{+} \pi^{0}$ exhibits neither an $\mathrm{ABC}$ effect nor a narrow resonance structure $[22,14]$ in this particular energy region (black filled circles in Fig. 3) - in agreement with the observations in all other two-pion channels of $p p$ initiated reactions $[15,16$, $17,18,19,20,21,23,24,25,26]$ and properly reproduced by conventional calculations of the $t$-channel $\Delta \Delta$ process $[22,14]$.

On the contrary the purely isoscalar fusion reaction $p n \rightarrow d \pi^{0} \pi^{0}$ does not behave as expected from conventional reaction dynamics. It rather exhibits a narrow resonance structure in the total cross section (blue filled circles in Fig. 3), which is correlated with the appearance of the ABC effect in the $\pi^{0} \pi^{0}$ invariant mass spectrum (Fig. 1) [12, 13, 22]. Its peak energy is about $90 \mathrm{MeV}$ below the nominal $\Delta \Delta$ threshold of $2 m_{\Delta}$ and its width of only $70 \mathrm{MeV}$ is much less than the width of $2 \Gamma_{\Delta}$ expected from the conventional $t$-channel $\Delta \Delta$ process. From the angular distributions the quantum numbers $I\left(J^{P}\right)=0\left(3^{+}\right)$have been assigned to this structure [12]. From Dalitz plots we may deduce that this state decays first into a $\Delta \Delta$ system before it reaches the $d \pi^{0} \pi^{0}$ final system.

Such kind of dibaryonic state has actually been predicted in some theoretical models, see e.g. $[36,37]$. At present no conventional process is known, which could at least qualitatively explain this phenomenon.

In the double-pionic fusion reaction $d d \rightarrow{ }^{4} \mathrm{He} \pi^{0} \pi^{0}$ again the $\mathrm{ABC}$ effect, which exibits a narrower and more pronounced enhancement than in the basic case - see Fig. 1 and 2, is observed to be correlated with the appearance of a resonance-like structure in the total cross section at the same excess energy, however, with an increased width due to Fermi motion in initial and final nuclei as well as due to collision damping, see Fig. 4 [30, 31]. From this we conclude that this resonance structure obviously is strong enough to survive even in light nuclei. 


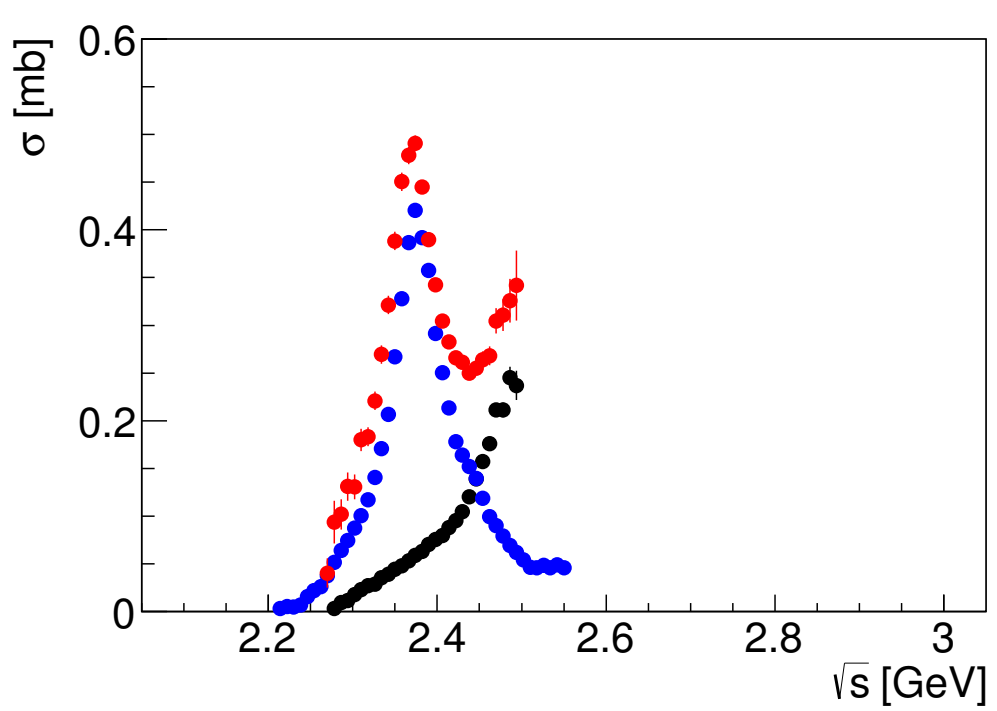

Figure 3: Total cross sections of the basic double-pionic fusion reactions $p N \rightarrow d \pi \pi$ of different isospin systems in dependence of the center-of-mass energy $\sqrt{s}$ from threshold $(\sqrt{s}=2.15 \mathrm{GeV})$ up to $2.6 \mathrm{GeV}$. The data for the isospin mixed reaction $p n \rightarrow d \pi^{+} \pi^{-}$are shown by the red full circles. The data for its isovector part given by half the cross section of the $p p \rightarrow d \pi^{+} \pi^{0}$ reaction are plotted by the black filled circles, whereas the data for its isoscalar part given by twice the cross section of the $p n \rightarrow d \pi^{0} \pi^{0}$ reaction are shown by the blue filled circles. From Ref. [35].

The energy dependence of the reaction $p d \rightarrow{ }^{3} \mathrm{He} \pi \pi$ is under investigation. Preliminary results $[38,39]$ indicate a similar trend as observed for the ${ }^{4} \mathrm{He}$ case. A pronunced $\mathrm{ABC}$ effect is observed at energies close to those, where the resonance is expected to reach its maximum and then it fades away at higher energies, where the $t$-channel $\Delta \Delta$ process becomes more relevant - see Fig. 5 .

\section{Conclusions and Outlook}

Based on the large amount of meanwhile available data we observe a scenario, which is surprisingly different, whether the two-pion production is initiated by isovector or isoscalar $N N$ collisions. Whereas all $p p$, i.e. isovector initiated reaction channels may be well understood by conventional $t$-channel processes, the two-pion production initiated by isoscalar $p n$ collisions is not understood by such processes alone. The outstanding finding here is that the $\mathrm{ABC}$ effect known for more than fifty years is strictly correlated with a narrow resonance-like structure in the total cross section. For the purely isoscalar $p n \rightarrow d \pi^{0} \pi^{0}$ reaction this phenomenon - interpreted as $s$-channel resonance with $I\left(J^{P}\right)=0\left(3^{+}\right)$- comprises most of the measured cross section. The same situation is found for the double-pionic fusion to the nuclei ${ }^{3} \mathrm{He}$ and ${ }^{4} \mathrm{He}$, thus this isoscalar $p n$ resonance structure is obviously strong enough to survive even in nuclei. In a more recent experiment the reaction channels $p n \rightarrow p n \pi^{0} \pi^{0}[40]$ and especially in $p n$ elastic scattering are being investigated in search of such a resonance. Also single-pion production channels could in principle contain effects from this resonance. However, the large conventional cross sections in these channels make it very difficult to search for the resonance there. 


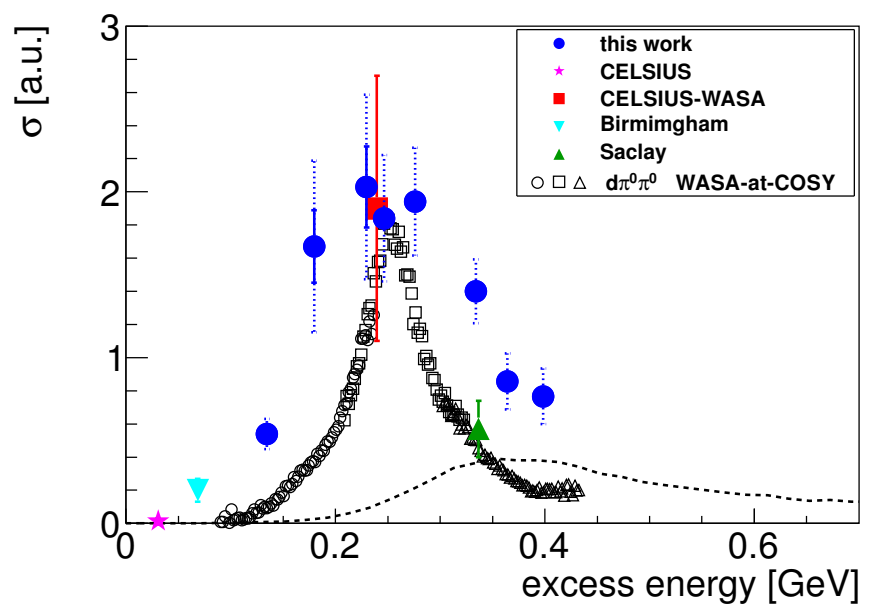

Figure 4: Total cross sections of the $p n \rightarrow d \pi^{0} \pi^{0}$ (black symbols) and $d d \rightarrow{ }^{4} \mathrm{He} \pi^{0} \pi^{0}$ reactions (colored symbols) in dependence of the excess energy above threshold. Both cross sections are given in relative units and scaled such as to have comparable heights in the peak cross section. The WASA-at-COSY results $[30,31]$ (solid circles) are compared to results from a previous exclusive measurement at CELSIUS/WASA [28] (square) as well as inclusive measurements at CELSIUS [41] (star), Birmingham [42] (inverted triangle) and Saclay [7] (triangle). The dotted line shows the energy dependence of the conventional $t$-channel $\Delta \Delta$ process, arbitrarily scaled in height. From Ref. [30, 31]
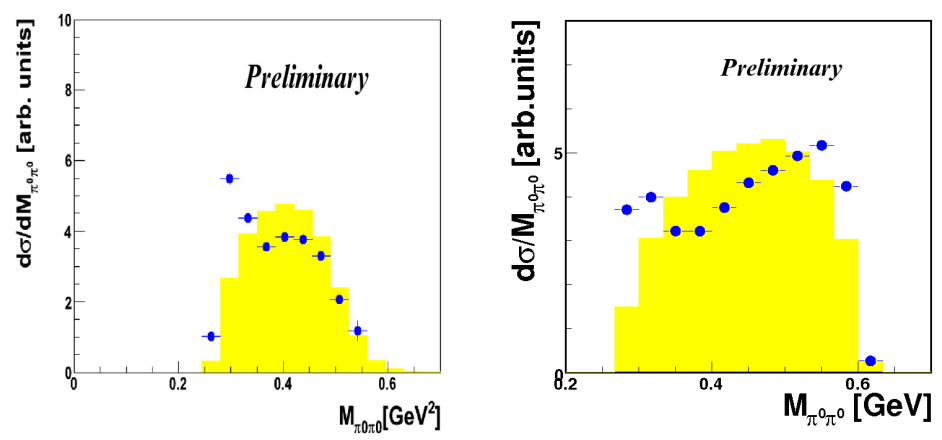

Figure 5: Distributions of the $\pi^{0} \pi^{0}$ invariant mass $M_{\pi^{0} \pi^{0}}$ observed in the reactions $d d \rightarrow{ }^{3} H e \pi^{0} \pi^{0}$ (left) and $p d \rightarrow{ }^{3} \mathrm{He} \pi^{0} \pi^{0}$ (right) at incident beam energies of $1.7 \mathrm{GeV}$ and $1.0 \mathrm{GeV}$ respectively, corresponding to $3.3 \mathrm{GeV} \leq \sqrt{s} \leq 3.41 \mathrm{GeV}$.. The shaded areas represent the phase-space distributions.

\section{Acknowledgments}

We acknowledge valuable discussions with C. Hanhart, E. Oset, A. Sibirtsev, F. Wang and C. Wilkin on this issue. This work has been supported by BMBF (06TU9193) and Forschungszentrum Jülich (COSY-FFE). 


\section{References}

[1] N. E. Booth, A. Abashian, K. M. Crowe, Phys. Rev. Lett. 7 (1961) 35; 5 (1960) 258; Phys. Rev. 132 (1963) $2296 \mathrm{ff}$

[2] I. Bar-Nir et al., Nucl. Phys. B 54 (1973) 17

[3] A. Abdivaliev et al., Sov. J. Nucl. Phys. 29 (1979) 796

[4] R. J. Homer et al., Phys. Rev. Lett. 9 (1964) 72

[5] J. H.Hall et al., Nucl. Phys. B 12 (1969) 573

[6] J. Banaigs et al., Nucl. Phys. B 67 (1973) 1

[7] J. Banaigs et al., Nucl. Phys. B 105 (1976) 52

[8] F.Plouin et al., Nucl. Phys. A 302 (1978) 413

[9] F. Plouin, P. Fleury, C. Wilkin, Phys. Rev. Lett. 65 (1990) 690

[10] R. Wurzinger et al., Phys. Lett. B 445 (1999) 423

[11] for a review see A. Codino and F. Plouin, LNS/Ph/94-06

[12] P. Adlarson et al., Phys. Rev. Lett. 106 (2011) 202302

[13] M. Bashkanov et al., Phys. Rev. Lett. 102 (2009) 052301

[14] F. Kren et al., Phys. Lett. B 684 (2010) 110 and B 702 (2011) 312; arXiv:0910.0995v2 [nucl-ex]

[15] J. Johanson et al., Nucl. Phys. A 712 (2002) 75

[16] W. Brodowski et al., Phys. Rev. Lett. 88 (2002) 192301

[17] J. Pätzold et al., Phys. Rev. C 67 (2003) 052202 (2005) 294

[18] T. Skorodko, et al., Eur. Phys. J. A 35 (2008) 317

[19] T. Skorodko, et al., Phys. Lett. B 679 (2009) 30

[20] T. Skorodko et al., Phys. Lett. B 695 (2011) 115

[21] T. Skorodko et al., Eur. Phs. J. A 47 (2011) 108

[22] P. Adlarson et al. Phys. Lett. B 721 (2013) 229;

[23] S. Abd El-Bary et al., Eur. Phys. J. A 37 (2008) 267

[24] S. Abd El-Samad et al., Eur. Phys. J. A 42 (2009) 159

[25] P. Adlarson et al., Phys. Lett. B 706 (2011) 256; arXiv:1107.0879 [hep-ex]

[26] S. Dymov et al., Phys. Rev. Lett. 102 (2009) 192301

[27] M. Bashkanov et al., Phys. Lett. B 637 (2006) 223

[28] S. Keleta et al., Nucl. Phys. A 825 (2009) 71

[29] F. Bellemann et al., Phys. Rev. C 60 (1999) 061002

[30] A. Pricking, PhD thesis, Univ. Tübingen 2011; http://tobias-lib.uni-tuebingen.de/volltexte/2011/5695/pdf/ThesisFinal.pdf

[31] P. Adlarson et al., Phys. Rev. C 87 (2013) 035204 Rapid Comm. 
[32] Ch. Bargholtz et al., Nucl. Instrum. Methods A 547 (2005) 294

[33] H. H. Adam et al., arXiv:nucl-ex/0411038 (2004)

[34] J. Bystricky it et al., J. Physique 48 (1987) 1901 and references therein

[35] COSY Annual Report highlights 2012

[36] J. Ping et al., Phys. Rev. C 79 (2009) 024001 and references therein

[37] P. J. Mulders, A. Th. Aerts and J. J. de Swart, Phys. Rev. D 21 (1980) 2653

[38] E. Perez del Rio et al., contribution to MESON12 conference, Cracow 2012, EPJ Web of Conferences 37,09029 (2012)

[39] E. Perez del Rio et al., contribution to the Etaprime Workshop, Aachen 2011; arXiv:1204.5509 [nucl-ex] (2012)

[40] G. Fäldt and C. Wilkin, Phys. Lett. B 701, (2011) 619

[41] Ch. Bargholtz et al., Phys. Lett. B 398 (1997) 264

[42] K. R. Chapman et al., Phys. Lett. 21 (1966) 465 